KEK-TH-766

UT-937

hep-th/0105129

\title{
Open String Fields As Matrices
}

\author{
Teruhiko Kawano \\ Department of Physics, University of Tokyo \\ Hongo, Tokyo 113-0033, Japan \\ kawano@hep-th.phys.s.u-tokyo.ac.jp \\ and \\ Kazumi Okuyama \\ Theory Group, KEK \\ Tsukuba, Ibaraki 305-0801, Japan \\ kazumi@post.kek.jp
}

We present a new representation of the string vertices of the cubic open string field theory. By using this three-string vertex, we attempt to identify open string fields as huge-sized matrices by following Witten's idea. By using these huge matrices, we obtain some results about the construction of partial isometries in the algebra of open string fields.

May, 2001 


\section{Introduction}

In the paper [1], Rastelli, Sen and Zwiebach have proposed the cubic open string field theory [2] around the tachyon vacuum, where it is supposed that only closed strings exist with no open strings. In the subsequent work [3], they found $D$-branes as classical solutions in their proposed open string field theory by using the projection fields obtained in [⿴囗十 for the matter sector. The analogous technique where projection operators are used to find classical solutions has also been seen in noncommutative field theories [5, 60. Especially in the latter paper [6], this solution generating technique has been used to find solutions in noncommutative gauge theory. This solution generating technique needs the system under consideration to have gauge invariance. Since the cubic open string field theory is invariant under huge gauge symmetry, it seems to be very useful in finding classical solutions analytically. In fact, the authors of [7],6] have argued the possibility that such techniques can be applied to the cubic string field theory and the boundary string field theory (See also [4] for another approach).

Let us briefly review their discussion about the application of the solution generating technique to the cubic string field theory. The variation of the action [2]

$$
S(A)=\frac{1}{g_{\mathrm{s}}^{2}} \operatorname{Tr}\left[\frac{1}{2} A \star Q A+\frac{1}{3} A \star A \star A\right],
$$

with respect to string field $A$, gives the equation of motion

$$
\frac{\delta S}{\delta A}=Q A+A \star A=0
$$

The action (1.1) is invariant under the infinitesimal gauge transformation

$$
-i \delta_{\Lambda} A=Q \Lambda+A \star \Lambda-\Lambda \star A
$$

and its finite form can be seen to be

$$
A \rightarrow A^{\prime}=U^{\dagger} \star Q U+U^{\dagger} \star A \star U
$$

where

$$
U=\exp _{\star}(-i \Lambda)=\sum_{n=0}^{\infty} \frac{(-i)^{n}}{n !} \underbrace{\Lambda \star \Lambda \star \cdots \star \Lambda}_{n \text { times }} .
$$

Now we turn to the solution generating technique with two operators, $U$ and $V$, satisfying

$$
U \star V=I, \quad V \star U=I-P,
$$


where $P$ is an operator such that $Q P=0$. Then we consider the field redefinition

$$
A^{\prime}=V \star Q U+V \star A \star U
$$

in the equation of motion (1.2) and obtain

$$
\frac{\delta S}{\delta A}\left(A^{\prime}\right)=V \star \frac{\delta S}{\delta A}(A) \star U
$$

which shows that if $A$ is a solution to (1.2), as far as $P \neq 0, A^{\prime}$ gives a new solution to it. The above condition $Q P=0$ is required to obtain (1.8), otherwise $A^{\prime}$ would no longer be a solution even if $A$ is a solution to the equation of motion (1.2). The simplest solution of (1.2) is $A=0$. Therefore, through the field redefinition (1.7), we can obtain another solution

$$
A_{c}=V \star Q U
$$

for any $U$ and $V$ satisfying (1.6). The action associated with the solution (1.9) turns out to be

$$
S\left(A_{c}\right)=-\frac{1}{6 g_{\mathrm{s}}^{2}} \operatorname{Tr}[V \star Q U \star V \star Q U \star V \star Q U] .
$$

If $D$-brane solutions could be obtained in this way, we could gain a deeper understanding of the tachyon condensation and the $D$-brane dynamics in the cubic string field theory.

In the cubic string field theory, our present technology does not seem enough to find such partial isometries $U, V$, let alone the nontrivial classical solutions to (1.2). In the case of the field theories [5, 6], under the Weyl transformation, fields are transformed to operators acting on the ordinary Hilbert space. Such operators can also be given as matrices in terms of states in the Hilbert space. Therefore, the Moyal product of fields is transformed to the usual matrix multiplication, which seems to make it easy to find projection operators and partial isometries. Thus, to develop analogous methods in the string field theory, we would be happy if we could have the formulation where open string fields can be treated as matrices. Recalling that the open string product $A \star B$ of fields $A$, $B$ is defined [2] such that the right half of the string of $A$ is identified with the left half of $B$ and summed over all the possible configurations to give the third string $A \star B$, we are very tempted to consider the right half of the string field $A$ as the column of some huge matrix and the left of $B$ as the row. In fact, Witten have already discussed the above idea in his paper [2]. In this paper, we will try to give an explicit realization of this idea by introducing a new representation of string vertices. Although we believe that our attempt 
is successful to some extent, there might exist some subtleties in our treatment of the midpoint of string vertices, as will be discussed in section 5 .

In section 2, we will present the new string vertices for the matter sector, which have a simpler form compared to the usual oscillator representation [8 12]. The kinetic term (the Virasoro operator) $L_{0}$ in a flat Minkowski spacetime becomes diagonal in terms of the usual oscillators of the string coordinates. In this paper, we introduce another set of oscillators. These oscillators do not any longer diagonalize the above Virasoro operator, but make string vertices very simple. Since our attention will be paid mainly to the open string product $\star$, the new oscillators will be adopted for our study in this paper. In addition, we have not found such simple vertices for the ghost sector. Since the open string product does not mix the ghost operators with ones in the matter sector, we will be content with the study of the open string product only in the matter sector.

In section 3, we will obtain the mapping from string fields to huge-sized matrices by using the above-mentioned three-string vertex. In section 4, taking advantage of the matrix representation of string fields, we will construct some projectors and partial isometries, and discuss the relation of these operators to $D$-branes. Most of section 5 is devoted to the discussion about potential subtleties in this work.

\section{A New Representation of String Vertices}

In this section, we shall introduce a new representation of the three-string vertex, which is given in terms of unusual oscillator modes of string coordinates. Although the BRS charge in a flat Minkowski spacetime is not diagonalized by the new oscillators, the three-string vertex becomes simple in terms of them. The simplicity of our string vertices turns out to give us more insights into the structure of the open string product.

\subsection{Preliminary}

To discuss the new representation of string vertices, we introduce unconventional oscillators in the Hilbert space of a single open string. Let us consider an open string parametrized by $\sigma \in[0, \pi]$ with the Neumann boundary condition at $\sigma=0, \pi$. The ordinary mode expansion of the coordinate $X^{i}(\sigma)$ and the momentum $P_{j}(\sigma)$ is given by

$$
\begin{aligned}
& X^{i}(\sigma)=x^{i}+i \sqrt{2 \alpha^{\prime}} \sum_{n \neq 0} \frac{1}{n} \alpha_{n}^{i} \cos n \sigma, \\
& P_{j}(\sigma)=\frac{1}{\pi}\left(p_{j}+\frac{1}{\sqrt{2 \alpha^{\prime}}} \sum_{n \neq 0} \eta_{j k} \alpha_{n}^{k} \cos n \sigma\right) .
\end{aligned}
$$


The canonical commutation relation is

$$
\left[X^{i}(\sigma), P_{j}\left(\sigma^{\prime}\right)\right]=i \delta_{j}^{i} \delta\left(\sigma, \sigma^{\prime}\right)
$$

where $\delta\left(\sigma, \sigma^{\prime}\right)$ is the $\delta$-function satisfying the Neumann boundary condition:

$$
\delta\left(\sigma, \sigma^{\prime}\right)=\frac{1}{\pi} \sum_{n=-\infty}^{\infty} \cos n \sigma \cos n \sigma^{\prime} .
$$

From (2.2), the commutation relations of the modes are found to be

$$
\left[x^{i}, p_{j}\right]=i \delta_{j}^{i}, \quad\left[\alpha_{n}^{i}, \alpha_{m}^{j}\right]=n \eta^{i j} \delta_{n+m, 0} .
$$

The $S L(2, \mathbf{R})$ vacuum $|0\rangle$ is defined by

$$
p_{j}|0\rangle=0, \quad \alpha_{n}^{i}|0\rangle=0 \quad(n \geq 1) .
$$

Now, let us introduce a new annihilation operator $a(\sigma)$ by

$$
a^{i}(\sigma)=\frac{1}{\sqrt{2}}\left(\sqrt{2 \pi \alpha^{\prime} t} P^{i}(\sigma)-i \frac{X^{i}(\sigma)}{\sqrt{2 \pi \alpha^{\prime} t}}\right),
$$

where $t$ is an arbitrary parameter 1 . Then, the commutator of $a^{i}(\sigma)$ and its hermitian conjugate $a_{j}^{\dagger}(\sigma)$ becomes

$$
\left[a^{i}(\sigma), a_{j}^{\dagger}\left(\sigma^{\prime}\right)\right]=\delta_{j}^{i} \delta\left(\sigma, \sigma^{\prime}\right)
$$

Using $a^{i}(\sigma)$, we define the " $a$-vacuum" $|\Omega\rangle$ by

$$
a^{i}(\sigma)|\Omega\rangle=0, \quad \text { for } \forall \sigma \in[0, \pi]
$$

It is well-known that the $S L(2, \mathbf{R})$ vacuum $|0\rangle$ and the $a$-vacuum $|\Omega\rangle$ are related by the Bogoliubov transformation. A set of functions $\left\{\varphi_{n}(\sigma)\right\}$ given on the interval $[0, \pi]$ by

$$
\varphi_{0}(\sigma)=\frac{1}{\sqrt{\pi}}, \quad \varphi_{n}(\sigma)=\sqrt{\frac{2}{\pi}} \cos n \sigma
$$

provide all the functions on $[0, \pi]$ with the Neumann boundary condition at $\sigma=0$, $\pi$ with a complete basis, in terms of which any of those functions can therefore be expanded. They are orthogonal on $[0, \pi]$ :

$$
\int_{0}^{\pi} d \sigma \varphi_{n}(\sigma) \varphi_{m}(\sigma)=\delta_{n, m}
$$

\footnotetext{
1 For the zero-mode part, the parameter $b$ in [3] corresponds to $4 \alpha^{\prime} t$.
} 
and are summed over to give the $\delta$-function

$$
\delta\left(\sigma, \sigma^{\prime}\right)=\sum_{n=0}^{\infty} \varphi_{n}(\sigma) \varphi_{n}\left(\sigma^{\prime}\right)
$$

The annihilation operator $a^{i}(\sigma)$ can thus be expanded by $\varphi_{n}$ as

$$
a^{i}(\sigma)=\sum_{n=0}^{\infty} a_{n}^{i} \varphi_{n}(\sigma)
$$

From (2.7), (2.11) and (2.12), we obtain the commutation relation

$$
\left[a_{n}^{i}, a_{m j}^{\dagger}\right]=\delta_{j}^{i} \delta_{n, m}
$$

Using (2.1) and (2.6), we can see that $a_{n}^{i}$ and $\alpha_{n}^{i}$ are related by

$$
\begin{aligned}
& a_{0}^{i}=\sqrt{\alpha^{\prime} t} p^{i}-i \frac{x^{i}}{2 \sqrt{\alpha^{\prime} t}} \\
& a_{n}^{i}=\frac{1}{2}\left(\sqrt{t}+\frac{1}{n \sqrt{t}}\right) \alpha_{n}^{i}+\frac{1}{2}\left(\sqrt{t}-\frac{1}{n \sqrt{t}}\right) \alpha_{-n}^{i} .
\end{aligned}
$$

By introducing the oscillators

$$
b_{n}^{i}=\frac{1}{\sqrt{n}} \alpha_{n}^{i}, \quad b_{n}^{i \dagger}=\frac{1}{\sqrt{n}} \alpha_{-n}^{i} \quad(n \geq 1)
$$

satisfying $\left[b_{n}^{i}, b_{m j}^{\dagger}\right]=\delta_{j}^{i} \delta_{n, m}$, the relation (2.14) can be rewritten as

$$
a_{n}^{i}=b_{n}^{i} \cosh \theta_{n}+b_{n}^{i \dagger} \sinh \theta_{n}
$$

where $\theta_{n}$ is given by

$$
e^{\theta_{n}}=\sqrt{t n}
$$

Therefore, $a_{n}^{i}$ and $b_{n}^{i}$ are related by the Bogoliubov transformation

$$
a_{n}^{i}=U b_{n}^{i} U^{-1}
$$

where $U$ is a unitary operator

$$
U=\exp \left(\frac{1}{2} \sum_{n=1}^{\infty} \theta_{n}\left(b_{n}^{2}-b_{n}^{\dagger 2}\right)\right)=\exp \left(\frac{1}{4} \sum_{n=1}^{\infty} \frac{\log t n}{n}\left(\alpha_{n}^{2}-\alpha_{-n}^{2}\right)\right)
$$


For the zero-mode part, the zero momentum eigenstate $|p=0\rangle$ and $a$-vacuum are related by

$$
|p=0\rangle=e^{-\frac{1}{2} a_{0}^{\dagger 2}}|\Omega\rangle .
$$

Note that the transformation in the zero-mode part is not unitary. After all, the relation between $|\Omega\rangle$ and $|0\rangle$ is found to be

$$
|\Omega\rangle=e^{\frac{1}{2} a_{0}^{\dagger 2}} U|0\rangle .
$$

\subsection{The String Vertices}

A string vertex is determined up to an overall normalization by an overlap condition. For the midpoint interaction as in Witten's open string field theory [2], the overlap condition is given by

$$
X_{r}^{i}(\sigma)-X_{r-1}^{i}(\pi-\sigma)=0, \quad P_{r}^{i}(\sigma)+P_{r-1}^{i}(\pi-\sigma)=0
$$

for $\sigma \in \mathrm{L}$, where $\mathrm{L}$ denotes the interval $[0, \pi / 2]$ and $\mathrm{R}$ will be used to denote $[\pi / 2, \pi]$. From (2.6), we find the overlap condition

$$
a_{r}^{i}(\sigma)=-a_{r-1}^{i}{ }^{\dagger}(\pi-\sigma) \equiv-r\left(a_{r-1}^{i}{ }^{\dagger}\right)(\sigma) \quad \text { for } \sigma \in \mathrm{L} .
$$

For the notational simplicity, we henceforth omit vector indices $i, j$ of the Lorentz group.

The overlap condition (2.23) is solved by the $N$-string vertex

$$
\begin{aligned}
& \left|v_{N}^{m}(1, \cdots, N)\right\rangle=e^{-V_{N}}|\Omega\rangle_{1 \ldots N} ; \\
& V_{N}=\sum_{r=1}^{N} \int_{0}^{\frac{\pi}{2}} d \sigma a_{r}^{\dagger}(\sigma) a_{r-1}^{\dagger}(\pi-\sigma) \\
& \quad=\frac{1}{2} \sum_{r, s=1}^{N} \int_{0}^{\pi} d \sigma d \sigma^{\prime} a_{r}^{\dagger}(\sigma) N^{r s}\left(\sigma, \sigma^{\prime}\right) a_{s}^{\dagger}\left(\sigma^{\prime}\right) .
\end{aligned}
$$

Here, the definition of the function $N^{r s}\left(\sigma, \sigma^{\prime}\right)$ is

$$
N^{r s}\left(\sigma, \sigma^{\prime}\right)=\left[\delta_{r-1, s} \theta_{\mathrm{L}}(\sigma)+\delta_{r+1, s} \theta_{\mathrm{R}}(\sigma)\right] \delta\left(\pi-\sigma, \sigma^{\prime}\right),
$$

where $\theta_{\mathrm{L}}(\sigma)$ and $\theta_{\mathrm{R}}(\sigma)$ are step functions with their supports $[0, \pi / 2]$ and $[\pi / 2, \pi]$, respectively. More precisely, the Fourier expansion of the step functions $\theta_{\mathrm{L}, \mathrm{R}}(\sigma)$ is given by

$$
\theta_{\mathrm{L}, \mathrm{R}}(\sigma)=\frac{1}{2} \pm \frac{2}{\pi} \sum_{n=1}^{\infty} \frac{1}{n} \sin \left(\frac{\pi}{2} n\right) \cos (n \sigma)
$$


Incidentally, by using the notation in (2.23), we can express $V_{N}$ as

$$
V_{N}=\sum_{r=1}^{N} \int_{\mathrm{L}} a_{r}^{\dagger} r\left(a_{r-1}^{\dagger}\right)
$$

where $\int_{\mathrm{L}}$ means $\int_{0}^{\frac{\pi}{2}} d \sigma$. The vertices 2.27 thus have very simple forms compared to the usual oscillator representation [8 12].

Before going further, let us make a small digression on a few computations to show how our vertices facilitate the computations which have been supposed to be very tedious. As the first computation, we pick up the sewing procedure of two string vertices by using the reflector $\langle R(I, J)|$ to obtain one string vertex. Indeed, we shall prove the equation

$$
\left\langle R(K, L)|| v_{M+1}^{m}(1, \cdots, M, K)\right\rangle\left|v_{N+1}^{m}(L, M+1, \cdots, M+N)\right\rangle=\left|v_{M+N}^{m}(1, \cdots, M+N)\right\rangle .
$$

The reflector $\langle R(I, J)|$ satisfies the defining condition $\langle R(1,2)| a_{r}(\sigma)=-\langle R(1,2)| r\left(a_{r-1}^{\dagger}\right)(\sigma)$ and is found to be

$$
\begin{aligned}
\langle R(1,2)| & ={ }_{12}\langle\Omega| \exp \left[-\sum_{r=1}^{2} \int_{0}^{\frac{\pi}{2}} d \sigma a_{r}(\sigma) a_{r-1}(\pi-\sigma)\right] \\
& ={ }_{12}\langle\Omega| \exp \left[-\int_{0}^{\pi} d \sigma a_{1}(\sigma) a_{2}(\pi-\sigma)\right] .
\end{aligned}
$$

By virtue of the formula $\left\langle\Omega\left|e^{\lambda \cdot a} e^{a^{\dagger} \cdot \mu}\right| \Omega\right\rangle=e^{\lambda \cdot \mu}$, where $\lambda \cdot \mu=\int_{0}^{\pi} d \sigma \lambda(\sigma) \mu(\sigma)$, we can see that

$$
\begin{aligned}
& \left\langle R(K, L)|| v_{M+1}^{m}(1, \cdots, M, K)\right\rangle\left|v_{N+1}^{m}(L, M+1, \cdots, M+N)\right\rangle \\
& \sim{ }_{K L}\left\langle\Omega\left|e^{-a_{K} \cdot r\left(a_{L}\right)} e^{-\int_{L}\left[a_{K}^{\dagger} r\left(a_{M}^{\dagger}\right)+a_{1}^{\dagger} r\left(a_{K}^{\dagger}\right)\right]} e^{-\int_{L}\left[a_{M+1}^{\dagger} r\left(a_{L}^{\dagger}\right)+a_{L}^{\dagger} r\left(a_{M+N}^{\dagger}\right)\right]}\right| \Omega\right\rangle_{K L} \\
& ={ }_{L}\left\langle\Omega\left|e^{\int_{L}\left[r\left(a_{M}^{\dagger}\right) r\left(a_{L}\right)+a_{1}^{\dagger} a_{L}\right]} e^{-\int_{L}\left[a_{M+1}^{\dagger} r\left(a_{L}^{\dagger}\right)+a_{L}^{\dagger} r\left(a_{M+N}^{\dagger}\right)\right]}\right| \Omega\right\rangle_{L} \\
& =e^{-\int_{L}\left[a_{M+1}^{\dagger} r\left(a_{M}^{\dagger}\right)+a_{1}^{\dagger} r\left(a_{M+N}^{\dagger}\right)\right]},
\end{aligned}
$$

which proves (2.28).

The next computation is concerned with the 'identity' field

$$
\langle I(K)|=\langle\Omega| \exp \left[-\frac{1}{2} a_{K} \cdot r\left(a_{K}\right)\right]
$$

satisfying the overlap condition

$$
\langle I(K)| a_{K}^{\dagger}(\sigma)=-\langle I(K)| r\left(a_{K}\right)(\sigma)
$$


for the $K$-th string. The use of the identity field allows us to obtain

$$
\left\langle I(N)|| v_{N}^{m}(1, \cdots, N-1, N)\right\rangle=\left|v_{N-1}^{m}(1, \cdots, N-1)\right\rangle .
$$

In order to prove this identity, the coherent state

$$
\begin{aligned}
& a(\sigma)|z\rangle=z(\sigma)|z\rangle \\
& |z\rangle=\exp \left[\int_{0}^{\pi} d \sigma a^{\dagger}(\sigma) z(\sigma)\right]|\Omega\rangle
\end{aligned}
$$

will be useful. Here $z(\sigma)$ is expanded as $z(\sigma)=\sum_{r=0}^{\infty} z_{n} \varphi_{n}(\sigma)$. Also, its hermitian conjugate $\langle z|$ satisfies

$$
\langle z| a^{\dagger}(\sigma)=\langle z| \bar{z}(\sigma) .
$$

Note that $\langle z| \neq{ }_{1}\langle r(z)| \equiv\langle R(1,2) \mid z\rangle_{2}$. Then, we can verify

$$
\int[d z d \bar{z}]|z\rangle e^{-\int_{0}^{\pi} d \sigma|z(\sigma)|^{2}}\langle z|=1
$$

where the measure is given by $[d z d \bar{z}]=\prod_{n=0}^{\infty} d x_{n} d y_{n}$ if $z_{n}=x_{n}+i y_{n}$.

By making use of the coherent state, we can prove the formula

$$
\begin{aligned}
& \exp \left[\frac{1}{2} a \cdot M \cdot a+\lambda \cdot a\right] \exp \left[\frac{1}{2} a^{\dagger} \cdot N \cdot a^{\dagger}+\mu \cdot a^{\dagger}\right]|\Omega\rangle \\
& =\sqrt{\operatorname{det}[1-M N]} \exp \left[-\frac{1}{2} \xi \cdot \mathcal{M}^{-1} \cdot \xi\right] \\
& \quad \exp \left[(\lambda \cdot N+\mu) \cdot(1-M N)^{-1} \cdot a^{\dagger}+\frac{1}{2} a^{\dagger} \cdot N(1-M N)^{-1} \cdot a^{\dagger}\right]|\Omega\rangle,
\end{aligned}
$$

where

$$
\begin{aligned}
& \mathcal{M}=\left(\begin{array}{cc}
M\left(\sigma, \sigma^{\prime}\right) & -\delta\left(\sigma, \sigma^{\prime}\right) \\
-\delta\left(\sigma, \sigma^{\prime}\right) & N\left(\sigma, \sigma^{\prime}\right)
\end{array}\right), \\
& \mathcal{M}^{-1}=-\left(\begin{array}{cc}
N(1-M N)^{-1}\left(\sigma, \sigma^{\prime}\right) & (1-N M)^{-1}\left(\sigma, \sigma^{\prime}\right) \\
(1-M N)^{-1}\left(\sigma, \sigma^{\prime}\right) & M(1-N M)^{-1}\left(\sigma, \sigma^{\prime}\right)
\end{array}\right),
\end{aligned}
$$

and $\xi(\sigma)=(\lambda(\sigma), \mu(\sigma))$.

The formula (2.37) helps us to calculate

$$
\begin{aligned}
& \left\langle I(N) \| v_{N}^{m}(1, \cdots, N-1, N)\right\rangle \\
& \sim{ }_{N}\left\langle\Omega\left|e^{-\frac{1}{2} a_{N} \cdot r\left(a_{N}\right)} e^{-\int_{\mathrm{L}}\left[a_{N}^{\dagger} r\left(a_{N-1}^{\dagger}\right)+a_{1}^{\dagger} r\left(a_{N}^{\dagger}\right)\right]}\right| \Omega\right\rangle_{N} \\
& =e^{-\int_{\mathrm{L}} a_{1}^{\dagger} r\left(a_{N-1}^{\dagger}\right)} .
\end{aligned}
$$


Thus, it completes the proof of the identity (2.33).

We conclude this section with the definition of the open string product. Given two string fields $A$ and $B$, we define the open string product $A \star B$ as

$$
|A \star B\rangle_{1}={ }_{3}\left\langle\left. r(A)\right|_{2}\left\langle r(B)|| v_{3}^{m}(1,2,3)\right\rangle,\right.
$$

recalling that ${ }_{1}\langle r(A)|=\langle R(1,2) \mid A\rangle_{2}$. For two coherent states $|z\rangle$ and $|w\rangle$, a small computation shows that the product $|z \star w\rangle$ is given by

$$
|z \star w\rangle=e^{-\int_{\mathrm{L}} r(z) \cdot w} e^{\int_{\mathrm{L}}\left(z a_{1}^{\dagger}+r(w) r\left(a_{1}^{\dagger}\right)\right)}|\Omega\rangle .
$$

If the notation $\left|z_{\mathrm{L}}, z_{\mathrm{R}}\right\rangle$ denotes $|z\rangle$ with the functions $z_{\mathrm{L}, \mathrm{R}}(\sigma)=\theta_{\mathrm{L}, \mathrm{R}}(\sigma) z(\sigma)$, the above equation (2.41) has a simple form

$$
\left|z_{\mathrm{L}}, z_{\mathrm{R}}\right\rangle \star\left|w_{\mathrm{L}}, w_{\mathrm{R}}\right\rangle=e^{-\int_{\mathrm{L}} r(z) \cdot w}\left|z_{\mathrm{L}}, w_{\mathrm{R}}\right\rangle
$$

\section{String Field Algebra}

\subsection{String Field Oscillators}

In this section, we construct the Fock space in which all the states are obtained by acting on the Fock vacuum $\left.\left|\Omega_{\text {str }}\right\rangle\right\rangle$ with string fields $A(\sigma)$ and $A^{\dagger}(\sigma)$. These string fields play the same role as the annihilation and creation operators, and satisfy the commutation relation

$$
\left[A(\sigma), A^{\dagger}\left(\sigma^{\prime}\right)\right]_{\star}=\delta\left(\sigma, \sigma^{\prime}\right) I, \quad \text { for } \sigma, \sigma^{\prime} \in \mathrm{R},
$$

where $[,]_{\star}$ denotes the commutator with the open string product:

$$
[A, B]_{\star}=A \star B-B \star A
$$

and $I$ denotes the identity field. We henceforth call the operators $A(\sigma), A^{\dagger}(\sigma)$ the "string field oscillators".

To seek the string field oscillators, let us begin with the open string product of fields $I(z)$ given by

$$
|I(z)\rangle=\exp \left[\int_{0}^{\pi} d \sigma z(\sigma) a^{\dagger}(\sigma)\right]|I\rangle .
$$

Making use of (2.37), we find that

$$
I(z) \star I(w)=e^{-\int_{\mathrm{L}} w \cdot r(z)} I(z+w) .
$$


Taking the derivatives with respect to $z(\sigma)$ and $w(\sigma)$ in (3.4), we obtain

$$
\left[a^{\dagger}(\sigma) I, a^{\dagger}\left(\sigma^{\prime}\right) I\right]_{\star}=\left(\theta_{\mathrm{L}}(\sigma)-\theta_{\mathrm{R}}(\sigma)\right) \delta\left(\sigma, \pi-\sigma^{\prime}\right) I
$$

Since the L.H.S. of (3.5) is antisymmetric under the exchange of $\sigma$ and $\sigma^{\prime}$, we can see that the R.H.S. is equal to $-\left(\theta_{\mathrm{L}}\left(\sigma^{\prime}\right)-\theta_{\mathrm{R}}\left(\sigma^{\prime}\right)\right) \delta\left(\sigma, \pi-\sigma^{\prime}\right) I$. Therefore, we have

$$
\begin{aligned}
& {\left[\theta_{\mathrm{R}}(\sigma) a^{\dagger}(\sigma) I, \theta_{\mathrm{R}}\left(\sigma^{\prime}\right) a^{\dagger}\left(\sigma^{\prime}\right) I\right]_{\star}=0,} \\
& {\left[\theta_{\mathrm{R}}(\sigma) a^{\dagger}(\sigma) I,-\theta_{\mathrm{R}}\left(\sigma^{\prime}\right) a^{\dagger}\left(\pi-\sigma^{\prime}\right) I\right]_{\star}=\theta_{\mathrm{R}}(\sigma) \delta\left(\sigma, \sigma^{\prime}\right) \theta_{\mathrm{R}}\left(\sigma^{\prime}\right) I,} \\
& {\left[\theta_{\mathrm{R}}(\sigma) a^{\dagger}(\pi-\sigma) I, \theta_{\mathrm{R}}\left(\sigma^{\prime}\right) a^{\dagger}\left(\pi-\sigma^{\prime}\right) I\right]_{\star}=0 .}
\end{aligned}
$$

The string field oscillators can thus be identified as

$$
A(\sigma)=\theta_{\mathrm{R}}(\sigma) a^{\dagger}(\sigma) I, \quad A^{\dagger}(\sigma)=-\theta_{\mathrm{R}}(\sigma) r\left(a^{\dagger}\right)(\sigma) I
$$

Here we notice that the identity $I$ maps the right part of the worldsheet oscillator $a^{\dagger}(\sigma)$ into the string field annihilation operator $A(\sigma)$ and the left part into the creation operator $A^{\dagger}(\sigma)$. Note that the form of $A(\sigma)$ is somehow reminiscent of the nilpotent string field $Q_{\mathrm{L}} I[13]$, where $Q$ is the BRS charge.

After the substitution $z(\sigma) \rightarrow z(\sigma) \theta_{\mathrm{R}}(\sigma)$ and $w(\sigma) \rightarrow w(\sigma) \theta_{\mathrm{R}}(\sigma)$ in (3.4), the derivatives at $z(\sigma)=w(\sigma)=0$ with respect to both of $z(\sigma)$ and $w(\sigma)$ give

$$
\begin{aligned}
& A(\sigma) \star A\left(\sigma^{\prime}\right)=\theta_{\mathrm{R}}(\sigma) a^{\dagger}(\sigma) \theta_{\mathrm{R}}\left(\sigma^{\prime}\right) a^{\dagger}\left(\sigma^{\prime}\right) I, \\
& A^{\dagger}(\sigma) \star A^{\dagger}\left(\sigma^{\prime}\right)=\theta_{\mathrm{R}}(\sigma) r\left(a^{\dagger}\right)(\sigma) \theta_{\mathrm{R}}\left(\sigma^{\prime}\right) r\left(a^{\dagger}\right)\left(\sigma^{\prime}\right) I .
\end{aligned}
$$

The recursive use of (3.8) provides

$$
\begin{aligned}
& \exp _{\star}\left(\int_{0}^{\pi} d \sigma w(\sigma) A^{\dagger}(\sigma)\right)=e^{-\int_{\mathrm{R}} w \cdot r\left(a^{\dagger}\right)} I, \\
& \exp _{\star}\left(\int_{0}^{\pi} d \sigma \bar{z}(\sigma) A(\sigma)\right)=e^{\int_{\mathrm{R}} \bar{z} \cdot a^{\dagger}} I .
\end{aligned}
$$

Here we have defined the exponential of string field $M$ by

$$
\exp _{\star}(M) \equiv \sum_{n=0}^{\infty} \frac{1}{n !} \underbrace{M \star M \star \cdots \star M}_{n \text { times }} .
$$

Taking a step further, we look for the Fock vacuum $\left.\left|\Omega_{\text {str }}\right\rangle\right\rangle$ of the string field oscillators;

$$
\left.A(\sigma)\left|\Omega_{\text {str }}\right\rangle\right\rangle=0
$$


To this end, it is appropriate to consider the open string product

$$
\begin{aligned}
& \left|w_{\mathrm{L}}, w_{\mathrm{R}}\right\rangle \star|I(z)\rangle=e^{-\int_{\mathrm{R}} w \cdot r(z)}\left|w_{\mathrm{L}}, w_{\mathrm{R}}+z_{\mathrm{R}}\right\rangle, \\
& |I(z)\rangle \star\left|w_{\mathrm{L}}, w_{\mathrm{R}}\right\rangle=e^{-\int_{\mathrm{R}} z \cdot r(w)}\left|z_{\mathrm{L}}+w_{\mathrm{L}}, w_{\mathrm{R}}\right\rangle .
\end{aligned}
$$

In (3.12), replacing $z(\sigma)$ with $z(\pi-\sigma) \theta_{\mathrm{L}}(\sigma)$ in the first equation and with $z(\sigma) \theta_{\mathrm{R}}(\sigma)$ in the second, we take the first derivative with respect to $z(\sigma)$ at $z(\sigma)=w(\sigma)=0$. Then we find that

$$
|\Omega\rangle \star A^{\dagger}(\sigma)=0, \quad A(\sigma) \star|\Omega\rangle=0,
$$

which, together with (3.11), indicates that

$$
\left.|\Omega\rangle \sim\left|\Omega_{\mathrm{str}}\right\rangle\right\rangle\left\langle\left\langle\Omega_{\mathrm{str}}\right|,\right.
$$

recalling here that $|\Omega\rangle$ is the $a$-vacuum in (2.8). Furthermore, combining (3.9) and (3.12), we find

$$
\begin{aligned}
& \left|w_{\mathrm{L}}, w_{\mathrm{R}}\right\rangle \star\left(e_{\star} \int_{\mathrm{R}} z \cdot A\right)=\left|w_{\mathrm{L}}, w_{\mathrm{R}}+z_{\mathrm{R}}\right\rangle, \\
& \left(e_{\star}-\int_{\mathrm{R}} r(z) \cdot A^{\dagger}\right) \star\left|w_{\mathrm{L}}, w_{\mathrm{R}}\right\rangle=\left|z_{\mathrm{L}}+w_{\mathrm{L}}, w_{\mathrm{R}}\right\rangle .
\end{aligned}
$$

From these equations (3.15), we understand that the coherent field $|z\rangle$ can be written as

$$
\begin{aligned}
\left|z_{\mathrm{L}}, z_{\mathrm{R}}\right\rangle & =\left(e_{\star}-\int_{\mathrm{R}} r(z) \cdot A^{\dagger}\right) \star|\Omega\rangle \star\left(e_{\star} \int_{\mathrm{R}} z \cdot A\right) \\
& \left.=\left(e_{\star}-\int_{\mathrm{R}} r(z) \cdot A^{\dagger}\right)\left|\Omega_{\mathrm{str}}\right\rangle\right\rangle\left\langle\left\langle\Omega_{\mathrm{str}}\right|\left(e_{\star} \int_{\mathrm{R}} z \cdot A\right) .\right.
\end{aligned}
$$

Note that the open string product $\star$ in the first line is replaced by the usual matrix multiplication in the second line in (3.16).

As a simple consistency check, we can verify that the equation (2.42) can also be retrieved by using the representation in the R.H.S. of (3.16) with the help of the formula

$$
\left\langle\left\langle\Omega_{\mathrm{str}}\left|\left(e_{\star} \int_{\mathrm{R}} z \cdot A\right)\left(e_{\star}-\int_{\mathrm{R}} r(w) \cdot A^{\dagger}\right)\right| \Omega_{\mathrm{str}}\right\rangle\right\rangle=e^{-\int_{\mathrm{L}} r(z) \cdot w} .
$$

It is convenient to introduce the string field coherent state $|z\rangle\rangle$ and its conjugate such that

$$
\left.|z\rangle\rangle=\left(e_{\star} \int_{\mathrm{R}} z \cdot A^{\dagger}\right)\left|\Omega_{\mathrm{str}}\right\rangle\right\rangle, \quad\left\langle\langle w|=\left\langle\left\langle\Omega_{\mathrm{str}}\right|\left(e_{\star} \int_{\mathrm{R}} \bar{w} \cdot A\right),\right.\right.
$$


satisfying that $\left.A(\sigma)|z\rangle\rangle=\theta_{\mathrm{R}}(\sigma) z(\sigma)|z\rangle\right\rangle,\left\langle\langle w| A^{\dagger}(\sigma)=\left\langle\langle w| \theta_{\mathrm{R}}(\sigma) \bar{w}(\sigma)\right.\right.$. Therefore, the coherent state in terms of these states becomes

$$
\left.|z\rangle=\left|z_{\mathrm{L}}, z_{\mathrm{R}}\right\rangle=|-r(z)\rangle\right\rangle\left\langle\left\langle\bar{z}|=|-r\left(z_{\mathrm{L}}\right)\right\rangle\right\rangle\left\langle\left\langle\bar{z}_{\mathrm{R}}\right|\right.
$$

and (3.17) turns into $\langle\langle\bar{z} \mid-r(w)\rangle\rangle=\exp \left(-\int_{\mathrm{R}} r(w) \cdot z\right)$.

We have so far seen that the open string product can be rephrased by using the ordinary matrix product. The 'integration' of the open string field theory is defined by using the identity field $|I\rangle$ as $\int\left|z_{\mathrm{L}}, w_{\mathrm{R}}\right\rangle=\left\langle I \mid z_{\mathrm{L}}, w_{\mathrm{R}}\right\rangle$, which is especially used in defining the action of the string field theory. In our matrix language, this integration can be seen to correspond to the trace

$$
\operatorname{Tr}(|z\rangle\rangle\langle\langle w|) \equiv\langle\langle w \mid z\rangle\rangle=\langle I \mid-r(z), \bar{w}\rangle
$$

Moreover, the inner product $\langle R(1,2)|| z\rangle_{1}|w\rangle_{2}$ can be given by the trace as

$$
\langle R(1,2)|| z\rangle_{1}|w\rangle_{2}=\langle r(z) \mid w\rangle=e^{-r(z) \cdot w}=\operatorname{Tr}(|-r(z)\rangle\rangle\langle\langle\bar{z}||-r(w)\rangle\rangle\langle\langle\bar{w}|) .
$$

\subsection{The Mode Expansion of the String Field Oscillators}

Since the worldsheet oscillator $a^{\dagger}(\sigma)$ is expanded as

$$
a^{\dagger}(\sigma)=\sum_{n=0}^{\infty} a_{n}^{\dagger} \varphi_{n}(\sigma)
$$

we also have the mode expansion of $A(\sigma)$ and $A^{\dagger}(\sigma)$ as

$$
A(\sigma)=\sum_{n=0}^{\infty} a_{n}^{\dagger} I \theta_{\mathrm{R}}(\sigma) \varphi_{n}(\sigma), \quad A^{\dagger}(\sigma)=-\sum_{n=0}^{\infty} a_{n}^{\dagger} I \theta_{\mathrm{R}}(\sigma) \varphi_{n}(\pi-\sigma)
$$

as seen from (3.7). In this subsection, we show that these string oscillators can be expanded as

$$
A(\sigma)=\sqrt{2} \sum_{n=0}^{\infty} A_{n} \varphi_{2 n}(\sigma), \quad A^{\dagger}(\sigma)=\sqrt{2} \sum_{n=0}^{\infty} A_{n}^{\dagger} \varphi_{2 n}(\sigma),
$$

in terms of $\left\{\varphi_{2 n}(\sigma)\right\}_{n=0,1, \ldots}$ instead of $\left\{\varphi_{n}(\sigma)\right\}_{n=0,1, \ldots}$ Although $A(\sigma)$ and $A^{\dagger}(\sigma)$ are defined on the interval $[\pi / 2, \pi]$, the operator $A(\sigma)$ can be extended to an operator $\mathcal{A}$ on the interval $[0, \pi]$ such that $\mathcal{A}(\sigma)=\theta_{\mathrm{R}}(\sigma) A(\sigma)+\theta_{\mathrm{L}}(\sigma) A(\pi-\sigma)$, and similarly for $\mathcal{A}^{\dagger}(\sigma)$. Because these operators $\mathcal{A}$ and $\mathcal{A}^{\dagger}$ satisfy the Neumann boundary condition, they are expanded in terms of $\left\{\varphi_{n}(\sigma)\right\}_{n=0,1, \ldots}$. However they also satisfy $\mathcal{A}(\sigma)=\mathcal{A}(\pi-\sigma)$ and are 
thus expanded by the even-integer modes $\left\{\varphi_{2 n}(\sigma)\right\}_{n=0,1, \ldots}$. The restriction of $\mathcal{A}(\sigma)$ and $\mathcal{A}^{\dagger}(\sigma)$ onto the interval $[\pi / 2, \pi]$ gives $(3.24)$.

The mapping of the modes $a_{n}^{\dagger} I$ to the modes $A_{n}$ and $A_{n}^{\dagger}$ can be obtained by using the relations

$$
A_{n}=\sqrt{2} \int_{\frac{\pi}{2}}^{\pi} d \sigma A(\sigma) \varphi_{2 n}(\sigma), \quad A_{n}^{\dagger}=\sqrt{2} \int_{\frac{\pi}{2}}^{\pi} d \sigma A^{\dagger}(\sigma) \varphi_{2 n}(\sigma),
$$

with the substitution of (3.23) and inversely

$$
\sum_{n=0}^{\infty} a_{n}^{\dagger} I \varphi_{n}(\sigma)=\theta_{\mathrm{R}}(\sigma) A(\sigma)-\theta_{\mathrm{L}}(\sigma) A^{\dagger}(\pi-\sigma)
$$

In fact, we obtain

$$
A_{n}=\sum_{m=0}^{\infty} R_{n m} a_{m}^{\dagger} I, \quad A_{n}^{\dagger}=\sum_{m=0}^{\infty} L_{n m} a_{m}^{\dagger} I
$$

where the definition of the matrices $R_{n m}$ and $L_{n m}$ is

$$
R_{n m}=\sqrt{2} \int_{\frac{\pi}{2}}^{\pi} d \sigma \varphi_{2 n}(\sigma) \varphi_{m}(\sigma), \quad L_{n m}=-\sqrt{2} \int_{0}^{\frac{\pi}{2}} d \sigma \varphi_{2 n}(\sigma) \varphi_{m}(\sigma)
$$

See appendix A for the properties of these matrices. The inverse mapping turns out to be

$$
a_{m}^{\dagger} I=\sum_{n=0}^{\infty}\left[A_{n} R_{n m}+A_{n}^{\dagger} L_{n m}\right]
$$

Thus, all the modes $A_{n}$ and $A_{n}^{\dagger}$ are almost in one-to-one correspondence with all the modes $a_{n}^{\dagger}$.

Let us consider the coherent state $|z\rangle$ in terms of the modes $A^{\dagger}(\sigma)$. Although the function $z(\sigma)$ in the coherent state $|z\rangle$ is defined on $[0, \pi]$ and has the expansion $z(\sigma)=\sum_{n=0}^{\infty} z_{n} \varphi_{n}(\sigma)$, its left and right parts $\theta_{\mathrm{L}, \mathrm{R}}(\sigma) z(\sigma)$ can be expanded as $\theta_{\mathrm{L}}(\sigma) z(\sigma)=\sqrt{2} \sum_{n=0}^{\infty} z_{\mathrm{L}, n} \varphi_{2 n}(\sigma)$ and $\theta_{\mathrm{R}}(\sigma) z(\sigma)=\sqrt{2} \sum_{n=0}^{\infty} z_{\mathrm{R}, n} \varphi_{2 n}(\sigma)$, in the same way as the operators $A(\sigma), A^{\dagger}(\sigma)$ in (3.24). Therefore, from (3.16), we obtain

$$
\left.\left|z_{\mathrm{L}}, z_{\mathrm{R}}\right\rangle=e_{\star}-\sum_{n=0}^{\infty} z_{\mathrm{L}, n} A_{n}^{\dagger}\left|\Omega_{\mathrm{str}}\right\rangle\right\rangle\left\langle\left\langle\Omega_{\mathrm{str}}\right| e_{\star} \sum_{n=0}^{\infty} z_{\mathrm{R}, n} A_{n}\right.
$$

2 In order to prove the statement, we would need to estimate the Jacobian associated with the change of the variables (3.27). From the condition $\mathcal{A}(\sigma)=\mathcal{A}(\pi-\sigma)$, we can see that $\mathcal{A}(\sigma)$ satisfies the extra boundary condition $\partial_{\sigma} \mathcal{A}(\sigma=\pi / 2)=0$. Therefore, the mapping (3.29): $A_{n}, A_{n}^{\dagger} \mapsto a_{n}^{\dagger}$ probably is not completely surjective. This subtlety will be discussed in section 5 . 
and the string field coherent state is thus rewritten as

$$
\left.|-r(z)\rangle\rangle=e_{\star}-\sum_{n=0}^{\infty} z_{\mathrm{L}, n} A_{n}^{\dagger}\left|\Omega_{\mathrm{str}}\right\rangle\right\rangle
$$

in terms of the modes $A_{n}^{\dagger}$. Making use of the modes $A_{n}^{\dagger}$, we can define states with definite occupation numbers of the modes:

$$
\left.|\mathbf{n}\rangle\rangle=\sqrt{\frac{1}{n_{0} ! n_{1} ! \cdots}}\left(A_{0}^{\dagger}\right)^{n_{0}}\left(A_{1}^{\dagger}\right)^{n_{1}} \cdots\left|\Omega_{\mathrm{str}}\right\rangle\right\rangle
$$

where the infinitely dimensional vector $\mathbf{n}$ denotes $\left(n_{0}, n_{1}, \cdots\right)$. In terms of such states $\left.|\mathbf{n}\rangle\right\rangle$, we have

$$
\left.|-r(z)\rangle\rangle=\sum_{n_{0}, n_{1}, \cdots=0}^{\infty}(-)^{\left(\sum_{i=0}^{\infty} n_{i}\right)} \frac{\left(z_{\mathrm{L}, 0}\right)^{n_{0}}\left(z_{\mathrm{L}, 1}\right)^{n_{1}} \cdots}{\sqrt{n_{0} ! n_{1} ! \cdots}}|\mathbf{n}\rangle\right\rangle .
$$

Since (3.25) and the commutation relation (3.1) provide the commutation relations among the modes $A_{n}$ and $A_{n}^{\dagger}$ as

$$
\left[A_{n}, A_{m}^{\dagger}\right]_{\star}=\delta_{n, m} I, \quad\left[A_{n}^{\dagger}, A_{m}^{\dagger}\right]_{\star}=\left[A_{n}, A_{m}\right]_{\star}=0
$$

the states $|\mathbf{n}\rangle\rangle$ satisfy the orthonormality condition

$$
\langle\langle\mathbf{m} \mid \mathbf{n}\rangle\rangle=\delta_{m_{0}, n_{0}} \delta_{m_{1}, n_{1}} \cdots \equiv \delta_{\mathbf{m}, \mathbf{n}}
$$

Since we have the mapping from string fields to matrices, a generic string field can be written as

$$
\begin{aligned}
|\Psi\rangle & =\int[d z d \bar{z}] e^{-z \cdot \bar{z}}|z\rangle\langle z \mid \Psi\rangle \\
& \left.=\int[d z d \bar{z}] e^{-z \cdot \bar{z}} \Psi(z)|-r(z)\rangle\right\rangle\langle\langle\bar{z}|,
\end{aligned}
$$

where $\Psi(z)=\langle z \mid \Psi\rangle$. In this way, all string fields can be converted to matrices on $\mathcal{H}_{s t r}$, which is spanned by the basis of the vectors $\{|\mathbf{n}\rangle\rangle\}$. In particular, the identity field $I$ is converted to

$$
\left.I=\int[d z d \bar{z}] e^{-z \cdot \bar{z}-\int_{\mathrm{L}} \bar{z} \cdot r(\bar{z})}|-r(z)\rangle\right\rangle\langle\langle\bar{z}| .
$$

Since $|-r(z)\rangle\rangle$ and $\langle\langle z|$ are expanded by $\mid \mathbf{n}\rangle\rangle$ and $\langle\langle\mathbf{m}|$, respectively, a generic string field $\Psi$ can be written as

$$
\left.\Psi=\sum_{\mathbf{n}, \mathbf{m}} \Psi_{\mathbf{n}, \mathbf{m}}|\mathbf{n}\rangle\right\rangle\langle\langle\mathbf{m}|
$$


Then for the identity field $I$, since $I \star \Psi=\Psi \star I=\Psi$ up to some potential anomalies [14,15, 16, we expect that the formula

$$
\left.I=\sum_{\mathbf{n}}|\mathbf{n}\rangle\right\rangle\langle\langle\mathbf{n}|
$$

holds. In order to prove $(\underline{3.39})$, we have to precisely estimate the measure $[d z d \bar{z}]$ in (3.37) under the change of the variables: $z_{n} \rightarrow z_{\mathrm{L}, n}, z_{\mathrm{R}, n}$, which we can perform in a similar way to the mapping (3.29). To this end, we need to compute the Jacobian associated with the change of the variables, but we have not done such a computation. Instead, we will hereafter assume (3.39).

\section{Some Applications of the String Field Algebra}

In the last section, we have seen that string fields can be expanded in terms of the operators $|\mathbf{n}\rangle\rangle\langle\langle\mathbf{m}|$. As explained in the lecture [17] (and related references therein), in the case of noncommutative (NC) $\mathbf{R}^{2}$, where we have the creation and annihilation operators $a^{\dagger}, a$ satisfying $\left[a, a^{\dagger}\right]=1$, the generating function

$$
G(z, \bar{z})=|z\rangle\left\langle z\left|=e^{z a^{\dagger}}\right| 0\right\rangle\left\langle 0\left|e^{\bar{z} a}=\sum_{n, m=0}^{\infty} \frac{z^{n} \bar{z}^{m}}{\sqrt{n ! m !}}\right| n\right\rangle\langle m|
$$

of the operators $|n\rangle\langle m|$ is a key ingredient to find projection operators and shift operators, of which the latter give a field theoretical analog of the partial isometries $U$ and $V$ mentioned in the introduction. Therefore, before proceeding to the string field theory, let us briefly recall some facts we shall need later.

The defining property of $G(z, \bar{z})$ is

$$
G(z, \bar{z}) \star G(w, \bar{w})=e^{\bar{z} w} G(z, \bar{w})
$$

and we impose on $G(z, \bar{z})$ the boundary condition

$$
G(0,0)=2 e^{-x^{2}}=|0\rangle\langle 0|
$$

The generating function $P(u)$ of projection operators $P_{n}=|n\rangle\langle n|$ :

$$
P(u)=\sum_{n=0}^{\infty} P_{n} u^{n}
$$


can be given by using $G(z, \bar{z})$ as

$$
\int \frac{d^{2} z}{\pi} e^{-|z|^{2}} G\left(u^{\frac{1}{2}} z, u^{\frac{1}{2}} \bar{z}\right)=P(u)
$$

In particular, since the generating function $P(u)$ satisfies $P(0)=P_{0}, P(1)=I$, the completeness condition

$$
\sum_{n=0}^{\infty} P_{n}=I
$$

can also be expressed by (4.5) at $u=1$;

$$
\int \frac{d^{2} z}{\pi} e^{-|z|^{2}} G(z, \bar{z})=I
$$

Moreover, using the function $G(z, \bar{z})$, we can construct the shift operator

$$
S=\sum_{n=0}^{\infty}|n+1\rangle\langle n|=\sum_{n=0}^{\infty} \sqrt{n !(n+1) !} \oint \frac{d z}{z^{n+2}} \oint \frac{d \bar{z}}{\bar{z}^{n+1}} G(z, \bar{z})
$$

\subsection{The Generating Function of the Operators $|\mathbf{n}\rangle\rangle\langle\langle\mathbf{m}|$}

We can see from the previous section that the generating function $G(z, w)$ of the string field theory is given by

$$
G(z, \bar{w})=|z\rangle\rangle\langle\langle w|
$$

which can indeed be verified from (3.17) to satisfy the stringy extension of the defining property $(4.2)$ :

$$
\begin{aligned}
G(z, \bar{z}) \star G(w, \bar{w}) & =e^{\int_{\mathrm{R}} \bar{z} \cdot w} G(z, \bar{w}) \\
& =e^{\sum_{n=0}^{\infty} \bar{z}_{\mathrm{R}, n} \cdot w_{\mathrm{R}, n}} G(z, \bar{w}),
\end{aligned}
$$

where $\theta_{\mathrm{R}}(\sigma) \bar{z}(\sigma)=\sqrt{2} \sum_{n=0}^{\infty} \bar{z}_{\mathrm{R}, n} \varphi_{2 n}(\sigma)$ and similarly for $w(\sigma)$. The equation (3.36) provides the counterpart of (4.7) for $u=1$;

$$
I=\int[d z d \bar{z}] e^{-z \cdot \bar{z}-\int_{\mathrm{L}} \bar{z} \cdot r(\bar{z})} G(-r(z), z) .
$$

\subsection{Projection States and Partial Isometries}

In the open string field theory around the tachyon vacuum [1], projection operators found in [4] have been used to describe $D$-branes as soliton solutions [3]. Before that, we had seen a somehow analogous usage of projection operators in the noncommutative field 
theory [5, 18, 19]. Therefore, it is natural to study projections in the algebra of string fields. The defining property of the projection state $|P\rangle$ is $P \star P=P$; more precisely,

$$
|P\rangle_{1}={ }_{3}\langle r(P)|{ }_{2}\left\langle r(P) \| v_{3}^{m}(1,2,3)\right\rangle \text {. }
$$

In general, for orthogonal projectors $P_{n}$ satisfying

$$
P_{n} \star P_{m}=\delta_{n, m} P_{m}, \quad \sum_{n=0}^{\infty} P_{n}=I,
$$

the generating function

$$
P(u)=\sum_{n=0}^{\infty} P_{n} u^{n}
$$

can be seen to satisfy the equation $P(u) \star P(v)=P(u v)$, to which one of the solutions is found to be

$$
P(u)=\exp \left(-\frac{u}{2} a^{\dagger} \cdot r\left(a^{\dagger}\right)\right)|\Omega\rangle
$$

Note that $P(u)$ interpolates between the $a$-vacuum and the identity

$$
P(0)=|\Omega\rangle, \quad P(1)=\exp \left(-\frac{1}{2} a^{\dagger} \cdot r\left(a^{\dagger}\right)\right)|\Omega\rangle=I .
$$

The projectors $P_{n}$ can thus be read as

$$
P_{n}=\frac{1}{n !}\left(-\frac{1}{2} a^{\dagger} \cdot r\left(a^{\dagger}\right)\right)^{n}|\Omega\rangle
$$

However, unfortunately these projection operators $P_{n}$ in (4.17) do not have finite norms; $\left\langle P_{n} \mid P_{n}\right\rangle=\infty$. Incidentally, $P(u)$ in the usual oscillator formalism [12] is presented in appendix B, and its norm also turns out to be ill-defined.

Besides the obvious example of the projectors $\left.P_{\mathbf{n}}=|\mathbf{n}\rangle\right\rangle\langle\langle\mathbf{n}|$, an alternative projector is given by $P(z)=e^{-\frac{1}{2} z \cdot r(z)}|z\rangle$, where the reality condition $\langle P|=\langle r(P)|$ enforces $z(\sigma)=$ $\sum_{n=0}^{\infty} z_{n} \varphi_{n}(\sigma)$ to satisfy that $\bar{z}_{n}=(-)^{n+1} z_{n}$. This projection operator is associated with the coherent state $|z\rangle\rangle$ as

$$
P(z)=\frac{|z\rangle\rangle\langle\langle z|}{\langle\langle z \mid z\rangle\rangle}=e^{\int_{\mathrm{R}}-z \bar{z}-z r\left(a^{\dagger}\right)+\bar{z} a^{\dagger}}|\Omega\rangle .
$$

The projection operator $\left.P_{\mathbf{0}}=\left|\Omega_{\text {str }}\right\rangle\right\rangle\left\langle\left\langle\Omega_{\text {str }}|=| \Omega\right\rangle\right.$ satisfies $\left.a(\sigma) \mid \Omega\right\rangle=0$, which suggests that $P_{\mathbf{0}}$ does not correspond to any extended objects 3 . Indeed, $P_{\mathbf{0}}=P(z=0)$ is an

3 The observation in this paragraph is mainly based on work in collaboration with Kazuki Ohmori [20]. 
instanton-like object, since its profile is found to be gaussian in all the directions, including the 'time' direction. Furthermore, the projector $P(z)$ can also be seen to be an instanton. Among the parameters $z_{n}$, the zero mode $z_{0}$ represents the location of the instanton $P(z)$ in spacetime. As in [1], if the ghost part of these solutions is universally given, the ratio of the norms of these solutions is equal to the ratio of their tensions in the tachyon string field theory [1]. Since the norm of the projector $P(z)$ is the same as that of $P_{\mathbf{0}}$, the moduli parameters $z(\sigma)$ may correspond to collective coordinates or the gauge redundancy. We shall leave a closer inspection of this problem to the future. The other projections $P_{\mathbf{n}}$ are also instanton-like. Thus, we may regard the projection operators $\sum_{k=1}^{N} P_{\mathbf{n}_{k}}$ as $N$ instanton solutions, though we have not fully uncovered the physics of such objects.

Finally, we shall discuss partial isometries in the cubic string field theory in our matrix language. As in [6, 17], our experience with noncommutative solitons on $\mathrm{NC}^{2}$ shows one obvious example of partial isometries

$$
U=\frac{1}{\sqrt{A_{n}^{\dagger} \star A_{n}+I}} \star A_{n}, \quad V=A_{n}^{\dagger} \star \frac{1}{\sqrt{A_{n}^{\dagger} \star A_{n}+I}} .
$$

The commutation relation $\left[A_{n}, A_{m}^{\dagger}\right]_{\star}=\delta_{n, m} I$ ensures that $U \star V=I$, by which we can verify that $V \star U$ is a projector. Since $\left.A_{n}\left|\Omega_{\text {str }}\right\rangle\right\rangle=0$, we can see that $V \star U \neq I$. These operators $U, V$ are thus the shift operators associated with the $n^{\text {th }}$ string field oscillator.

A further example can be given by

$$
\begin{aligned}
& \left.U=I-\sum_{n_{0}=0}^{\infty}\left|n_{0}, \mathbf{0}\right\rangle\right\rangle\left\langle\left\langle n_{0}, \mathbf{0}\left|+\sum_{n_{0}=0}^{\infty}\right| n_{0}, \mathbf{0}\right\rangle\right\rangle\left\langle\left\langle n_{0}+1, \mathbf{0}\right|,\right. \\
& \left.V=I-\sum_{n_{0}=0}^{\infty}\left|n_{0}, \mathbf{0}\right\rangle\right\rangle\left\langle\left\langle n_{0}, \mathbf{0}\left|+\sum_{n_{0}=0}^{\infty}\right| n_{0}+1, \mathbf{0}\right\rangle\right\rangle\left\langle\left\langle n_{0}, \mathbf{0}\right|,\right.
\end{aligned}
$$

with the defining property

$$
\left.U \star V=I, \quad V \star U=I-\left|\Omega_{\mathrm{str}}\right\rangle\right\rangle\left\langle\left\langle\Omega_{\mathrm{str}}\right| .\right.
$$

In this case, the pair $(U, V)$ is an analogue of the one used in the discussion of noncommutative $U(1)$ instantons on $\mathbf{R}^{4}[21]$.

Before concluding this section, we should point out that, in order for these partial isometries to give classical solutions, The action of the BRS charge $Q$ on the resulting projector $V \star U$ must vanish, as explained in the introduction. For the BRS charge $Q$ in a usual flat Minkowski spacetime, however, this turns out not to be the case. 


\section{Conclusion and Discussion}

In this paper, we have introduced a new presentation of the string vertices in the cubic string field theory. We have seen that the new string vertices have very simple structures and facilitate some computations which have been very complicated. By making use of these new vertices, we have tried to reformulate the open string field product or the $\star$ product as the usual matrix multiplication 1 . Indeed, we believe that to some extent in this paper, we have been successful in obtaining huge-sized matrices as open string fields. However, we have also been cavalier on two points: our treatment of the midpoint and some anomalies [14,15,16] associated with singularities of the $\star$-product.

Since our representation of the string vertices satisfies the overlap condition even at the midpoint, we believe there is nothing wrong with our string vertices. However, our discussion in section 3 about splitting the operator $a^{\dagger}(\sigma) I$ into $A_{n}$ and $A_{n}^{\dagger}$ is subtle, because of the usage of the functions $\theta_{\mathrm{L}, \mathrm{R}}(\sigma)$ as projectors; $\theta_{\mathrm{L}, \mathrm{R}}(\sigma)^{2}=\theta_{\mathrm{L}, \mathrm{R}}(\sigma)$. In addition, our Fourier transform $A_{n}, A_{n}^{\dagger}$ of the left and right halves of open strings does not seem enough to describe all the degrees of freedom of open strings, as mentioned in the footnote of section 3. Although, in the original suggestion [2], one additional degree of freedom associated with the midpoint of strings is added, it is not clear to us whether we should also introduce such a degree of freedom.

The string oscillators are defined by using the identity state $I$. It is known [16, 14 that states like $Q_{\mathrm{L}} I$ in 13 have anomalies in calculations with the string vertices. Since the modes $A_{n}$ and $A_{n}^{\dagger}$ are also defined by acting on $I$ with the integration of $a^{\dagger}(\sigma)$ over half of the open strings, there might exist some potential anomalies in the matrix multiplication in our formulation.

In this paper, we have restricted our attention to only the matter part of open strings. As for the ghost part, we have not been successful in finding a simple representation of the string vertices by using the fermionic ghosts $b(\sigma)$ and $c(\sigma)$. However, the ghost part of the vertices might be able to be simplified by using the bosonized ghost in a similar way to the matter part of our vertices. Since the construction of the ghost part requires the insertion of the ghosts at the midpoint, we would have more serious problems concerning the midpoint, as we have discussed in the above paragraphs.

4 In the original paper [2], Witten has already discussed such a possibility (see also [22, 23, 24] and for very recent discussions, [25,26]). 
We could have obtained our matrix formulation of the cubic open string field theory in the background $B$-field [27,28]. It would be interesting to explore what is happening in our matrix formulation in the large $B$ limit and to compare that with the discussion in [29,7].

As in the paper [3], it is found that the projectors of the matter part give the ratios between the $D$-brane solutions in the tachyon string field theory [1] with the ansatz of the universality of the ghost parts by making use of the method in [4]. It is possible to apply an analogous method to our vertices, and to find the projectors which are supposed to be $D$-branes. However, we can analytically calculate the norm of those solutions and find no finite tensions of those solutions except the instanton-like solutions given in section 4 . By the tensions we mean the norms of the solution divided by the volume factor of the corresponding $D$-brane worldvolume 5 . So far, we have not succeeded in finding any brane solutions with finite norms by using our three-string vertex. Since it is likely that the norm of such $D$-brane solutions can be computed analytically by using our vertex, the discovery of the solutions gives a nontrivial check on the interpretation of them as $D$-branes.

As discussed in [30], the charges of $D$-branes are classified by K-theory and it is closely related to the topological configurations of tachyon fields. Although $D$-branes in bosonic string theory do not carry any charges, it may be interesting to study K-theory of our matrix algebra [31] of string fields.

Note added: after completing this work, we received the papers [25,26] concerning a similar idea to ours: splitting open strings into their left and right halves. However, on the whole, our approach is slightly different from theirs [25,26], especially on the introduction of the new representation of the string vertices. As a technical point, upon splitting open strings, we used a different Fourier expansion of the 'left' and 'right' halves from theirs [25, 26]. It is at present unclear to us how these expansions are precisely related to each other.

Furthermore, from [25,26] we learnt of the references [22,23,24, where earlier discussions (also even earlier references therein) about the idea of splitting open strings are available, besides the original discussion in [2].

After submitting the original version of this paper to the arXiv.org e-print archive, we were informed of a paper [32] arguing another approach to formulate string fields as matrices.

5 This is also based on [20], as mentioned in section 4. 


\section{Acknowledgements}

The authors would like to thank Kazuki Ohmori for his collaboration at the early stage of this work and also for his careful reading of the manuscript and helpful suggestions on it. T.K. is deeply grateful to Taichiro Kugo and Hiroyuki Hata for helpful discussions. K. O. would like to thank Kazuyuki Furuuchi, Nobuyuki Ishibashi and Soo-Jong Rey for discussions, and to acknowledge the hospitality of theory group at Seoul National University in Korea. T. K. was supported in part by a Grant-in-Aid for Scientific Research in a Priority Area: "Supersymmetry and Unified Theory of Elementary Particles"(\#707), from the Ministry of Education, Culture, Sports, Science, and Technology. K. O. was supported in part by JSPS Research Fellowships for Young Scientists.

\section{Appendix A. Properties of $L_{n m}$ and $R_{n m}$}

To see the properties of the matrices $L_{n m}$ and $R_{n m}$ in (3.28), it is convenient to introduce other matrices $P_{\mathrm{L}}$ and $P_{\mathrm{R}}$ defined by

$$
\left(P_{\mathrm{L}}\right)_{n m}=\int_{\mathrm{L}} \varphi_{n} \varphi_{m}, \quad\left(P_{\mathrm{R}}\right)_{n m}=\int_{\mathrm{R}} \varphi_{n} \varphi_{m} .
$$

Since $\varphi_{n}$ transforms under the reflection as $r\left(\varphi_{n}\right)=(-1)^{n} \varphi_{n}, P_{\mathrm{L}}$ and $P_{\mathrm{R}}$ are related by

$$
P_{\mathrm{L}}=C P_{\mathrm{R}} C
$$

where $C_{n m}=(-1)^{n} \delta_{n m}$. By making use of the completeness condition (2.10), (2.11) of $\varphi_{n}$, we can see that $P_{\mathrm{L}}$ and $P_{\mathrm{R}}$ are orthogonal projections:

$$
P_{\mathrm{L}}+P_{\mathrm{R}}=1, \quad P_{\mathrm{L}, \mathrm{R}}^{2}=P_{\mathrm{L}, \mathrm{R}}, \quad P_{\mathrm{L}} P_{\mathrm{R}}=P_{\mathrm{R}} P_{\mathrm{L}}=0 .
$$

These relations can also be understood by noticing that $P_{\mathrm{L}, \mathrm{R}}$ are the representation matrices of $\theta_{\mathrm{L}, \mathrm{R}}(\sigma)$ on the basis $\left\{\varphi_{n}(\sigma)\right\}$

$$
\theta_{\mathrm{L}, \mathrm{R}}(\sigma) \varphi_{n}(\sigma)=\sum_{m=0}^{\infty}\left(P_{\mathrm{L}, \mathrm{R}}\right)_{n m} \varphi_{m}(\sigma) .
$$

From the definition of $R, L$ in (3.28), they are written in terms of $P_{\mathrm{R}, \mathrm{L}}$ as

$$
R_{n m}=\frac{1}{\sqrt{2}}\left[(1+C) P_{\mathrm{R}}\right]_{2 n, m}, \quad L_{n m}=-\frac{1}{\sqrt{2}}\left[(1+C) P_{\mathrm{L}}\right]_{2 n, m} .
$$


Using the relations (A.2) and (A.3), we obtain

$$
\begin{aligned}
& \sum_{k=0}^{\infty} R_{k n} R_{k m}=\frac{1}{2}\left[P_{\mathrm{R}}(1+C)^{2} P_{\mathrm{R}}\right]_{n m}=\left(P_{\mathrm{R}}\right)_{n m}, \\
& \sum_{k=0}^{\infty} L_{k n} L_{k m}=\frac{1}{2}\left[P_{\mathrm{L}}(1+C)^{2} P_{\mathrm{L}}\right]_{n m}=\left(P_{\mathrm{L}}\right)_{n m} .
\end{aligned}
$$

Therefore, $R$ and $L$ satisfy

$$
\sum_{k=0}^{\infty}\left(R_{k n} R_{k m}+L_{k n} L_{k m}\right)=\delta_{n m}
$$

This formula is used to show the relation (3.29) between $a_{n}^{\dagger}$ and $A_{n}, A_{n}^{\dagger}$. From the relation

$$
\int_{\mathrm{R}} \varphi_{2 n} \varphi_{2 m}=\int_{\mathrm{L}} \varphi_{2 n} \varphi_{2 m}=\frac{1}{2} \delta_{n m}
$$

we can also see that

$$
\sum_{k=0}^{\infty} R_{n k} R_{m k}=\sum_{k=0}^{\infty} L_{n k} L_{m k}=\delta_{n m}, \quad \sum_{k=0}^{\infty} R_{n k} L_{m k}=0 .
$$

\section{Appendix B. Generating Function of Projections using Gross-Jevicki Vertex}

In this appendix, we consider the generating function of orthogonal projections using the Gross-Jevicki vertex. We follow the notation in [3]. The function $P(u)$ satisfying $P(u) \star P(v)=P(u v)$ is found to be

$$
P(u)=\operatorname{det}^{\frac{1}{2}} \frac{(1-X)(1+T)}{T u+1} \exp \left(-\frac{1}{2} a^{\dagger} C T(u) a^{\dagger}\right)|0\rangle,
$$

where

$$
T(u)=\frac{u+T}{T u+1} .
$$

This can be checked by using the formula

$$
e^{-\frac{1}{2} a^{\dagger} C T_{1} a^{\dagger}}|0\rangle \star e^{-\frac{1}{2} a^{\dagger} C T_{2} a^{\dagger}}|0\rangle=\operatorname{det}^{-\frac{1}{2}}\left[1-X\left(T_{1}+T_{2}\right)+X T_{1} T_{2}\right] e^{-\frac{1}{2} a^{\dagger} C T_{12} a^{\dagger}}|0\rangle,
$$

where

$$
T_{12}=\frac{X-X\left(T_{1}+T_{2}\right)+T_{1} T_{2}}{1-X\left(T_{1}+T_{2}\right)+X T_{1} T_{2}} .
$$

Here we assumed that $C, T_{1}, T_{2}$ and $X$ commute with each other. Note that $P(u)$ interpolates between the matter part of "sliver" [15, 4, 3] and the identity:

$$
P(0)=|\infty\rangle_{m}, \quad P(1)=I .
$$




\section{References}

[1] L. Rastelli, A. Sen and B. Zwiebach, "String Field Theory Around the Tachyon Vacuum," hep-th/0012251.

[2] E. Witten, "Noncommutative Geometry And String Field Theory," Nucl. Phys. B268 (1986) 253.

[3] L. Rastelli, A. Sen and B. Zwiebach, "Classical Solutions in String Field Theory Around the Tachyon Vacuum," hep-th/0102112.

[4] V. A. Kostelecky and R. Potting, "Analytical construction of a nonperturbative vacuum for the open bosonic string," Phys. Rev. D63 (2001) 046007, hep-th/0008252.

[5] R. Gopakumar, S. Minwalla and A. Strominger, "Noncommutative Solitons," JHEP 0005 (2000) 020, hep-th/0003160.

[6] J. A. Harvey, P. Kraus and F. Larsen, "Exact Noncommutative Solitons," JHEP 0012 (2000) 024, hep-th/0010060.

[7] M. Schnabl, "String Field Theory at Large B-field and Noncommutative Geometry," JHEP 0011 (2000) 031, hep-th/0010034.

[8] S. Samuel, "The Physical and Ghost Vertices in Witten's String Field Theory," Phys. Lett. 181B (1986) 255.

[9] D. J. Gross and A. Jevicki, "Operator Formulation of Interacting String Field Theory," Nucl. Phys. B283 (1987) 1;

"Operator Formulation of Interacting String Field Theory (II)," Nucl. Phys. B287 (1987) 225.

[10] E. Cremmer, A. Schwimmer and C. Thorn, "The Vertex Function in Witten's Formulation of String Field Theory," Phys. Lett. 179B (1986) 57.

[11] K. Itoh, K. Ogawa and K. Suehiro, "BRS Invariance of Witten's Type Vertex," Nucl. Phys. B289 (1987) 127.

[12] N. Ohta, "Covariant Interacting String Field Theory in the Fock Space Representation," Phys. Rev. D 34 (1986) 3785 [Erratum-ibid. D 35 (1986) 2627].

[13] G. T. Horowitz, J. Lykken, R. Rohm and A. Strominger, "Purely Cubic Action for String Field Theory," Phys. Rev. Lett. 57 (1986) 283.

[14] G. T. Horowitz and A. Strominger, "Translations As Inner Derivations And Associativity Anomalies In Open String Field Theory," Phys. Lett. B185 (1987) 45.

[15] L. Rastelli and B. Zwiebach, "Tachyon potentials, star products and universality," hep-th/0006240.

[16] A. Strominger, "Point Splitting Regularization Of Classical String Field Theory," Phys. Lett. B187 (1987) 295.

[17] J. A. Harvey, "Komaba Lectures on Noncommutative Solitons and D-Branes," hepth/0102076. 
[18] K. Dasgupta, S. Mukhi and G. Rajesh, "Noncommutative Tachyons," JHEP 0006 (2000) 022, hep-th/0005006.

[19] J. A. Harvey, P. Kraus, F. Larsen and E. J. Martinec, "D-branes and Strings as Noncommutative Solitons," JHEP 0007 (2000) 042, hep-th/0005031.

[20] T. Kawano, K. Ohmori and K. Okuyama, unpublished.

[21] K. Furuuchi, "Topological Charge of U(1) Instantons," hep-th/0010006.

[22] H. Chan and S. T. Tsou, "String Theory Considered As A Local Gauge Theory Of An Extended Object," Phys. Rev. D 35, 2474 (1987);

"Yang-Mills Formulaton Of Interacting Strings," Phys. Rev. D 39, 555 (1989).

[23] J. Bordes, H. Chan, L. Nellen and S. T. Tsou, "Half String Oscillator Approach to String Field Theory," Nucl. Phys. B351 (1991) 441.

[24] A. Abdurrahman and J. Bordes, "The relationship between the comma theory and Witten's string field theory. I," Phys. Rev. D58 (1998) 086003.

[25] L. Rastelli, A. Sen and B. Zwiebach, "Half-strings, Projectors, and Multiple D-branes in Vacuum String Field Theory," hep-th/0105058.

[26] D. J. Gross and W. Taylor, "Split string field theory I," hep-th/0105059.

[27] T. Kawano and T. Takahashi, "Open String Field Theory on Noncommutative Space," Prog. Theor. Phys. 104 (2000) 459, hep-th/9912274;

"Open-Closed String Field Theory in the Background B-Field," Prog. Theor. Phys. 104 (2000) 1267, hep-th/0005080.

[28] F. Sugino, "Witten's Open String Field Theory in Constant B-Field Background," JHEP 0003 (2000) 017, hep-th/9912254.

[29] E. Witten, "Noncommutative Tachyons And String Field Theory," hep-th/0006071.

[30] E. Witten, "D-branes and K-theory," JHEP 9812 (1998) 019, hep-th/9810188.

[31] E. Witten, "Overview Of K-Theory Applied To Strings," Int. J. Mod. Phys. A16 (2001) 693, hep-th/0007175.

[32] I. Y. Arefeva and I. V. Volovich, "Two-dimensional gravity, string field theory and spin glasses," Phys. Lett. B 255, 197 (1991). 